\title{
A Simple and Flexible Bayesian Method for Inferring Step Changes in Cognition
}

\author{
Michael D. Lee \\ Department of Cognitive Sciences \\ University of California, Irvine
}

\begin{abstract}
Human behavioral data often shows patterns of sudden change over time. Sometimes the causes of these step changes are internal, such as learning curves changing abruptly when a learner implements a new rule. Sometimes the cause is external, such a people's opinions about a topic changing in response to a new relevant event. Detecting change points in sequences of binary data is a basic statistical problem, with many existing solutions, but they seem rarely to be used in psychological modeling. We develop a simple and flexible Bayesian approach to modeling step changes in cognition, implemented as a graphical model in JAGS. The model is able to infer how many change points are justified by the data, as well as the location of the change points. The basic model is also easily extended to include latent-mixture and hierarchical structures, allowing it to be tailored to specific cognitive modeling problems. We demonstrate the adequacy of the basic model by applying it to the classic Lindisfarne Scribes problem, and the flexibility of the modeling approach through two new applications. The first involves a latent-mixture model to determine if individuals learn categories incrementally or in discrete stages. The second involves a hierarchical model of crowd-sourced predictions about the winner of the U.S. National Football League's Most Valuable Player for the 2016-2017 season.
\end{abstract}

Keywords: step change, change points, change detection, Bayesian inference, spike-and-slab priors 


\section{Introduction}

All sorts of human behavior comes in the form of binary responses. People choose to press thumbs up or thumbs down, to book a window or an aisle seat, or to stay home for dinner or dine out. The rates of these binary responses usually change over time, and sometimes change suddenly and significantly. A scandal can see the rate of thumbs up responses for a celebrity or politician fall dramatically, a bad experience in a window seat can shift the preference to an aisle, and the probability of dining out may jump as the weekend approaches.

As these examples make clear, the statistical problem of detecting step changes in binary responses has applications throughout the cognitive sciences. There is a substantial statistical literature on methods for detecting change points. Many early methods relied on classic significance testing to find changes, using standard parametric and non-parametric tests (e.g., Pettitt, 1979; Venter \& Steel, 1996). Bayesian approaches were also developed relatively early. A. F. M. Smith (1975), for example, provided analytic Bayesian solution to a number of cases involving binomial and Gaussian distributions with a single change point. More recent Bayesian methods have relied on computational approaches to inference. One approach is to use general methods — such as reversible-jump Markov-chain Monte Carlo (MCMC) methods (Green, 1995), product partition methods (Barry \& Hartigan, 1993), discrete state Markov processes (Chib, 1998), and Gibbs sampling (Stephens, 1994) — and apply them to change-point inference as a special case of the more general capabilities they provide. Other computational Bayesian approaches have been specifically developed for change-point inference. For example, Fearnhead (2006) developed a clever recursive approach to MCMC computation that allows efficient inference for large problems, while Adams and MacKay (2007) developed a method for the on-line detection of change points.

These methods do not seem, however, to be widely used in cognitive modeling. In particular, computational Bayesian methods for the simultaneous inference of the number of change points and their location are rarely used. One barrier to their adoption may be difficulty of implementation, since the methods involve non-trivial algorithms. Another barrier may be the difficulty of adapting or extending published methods to specific psychological modeling problems. Unless the substantive psychological problem takes exactly the same statistical form as the existing solution which often involve technically convenient choices of simple sampling distributions 
and conjugate priors - additional theoretical and practical work is required. In this paper, we provide a Bayesian statistical approach to the multiple change-point problem that is designed to help address these barriers. Our approach has the practical attraction of having a simple implementation as a graphical model in standard JAGS software (Plummer, 2003). Because of this implementation, our approach also has the theoretical attraction of making it relatively easy to extend and adapt the basic change-point detection model to specific cognitive modeling problems.

The structure of this paper is as follows. We first present the basic statistical approach to inferring change points, and its implementation as a graphical model. We highlight the way in which the model combines Bayesian model selection and parameter inference through careful specification of prior distributions. After a simple demonstration with simulated data, we apply the model to the classic Lindisfarne Scribes problem (Silvey, 1958), showing that its inferences match those of existing methods. We then demonstrate the flexibility of the modeling approach in two case studies. The first case study involves human category learning data, and uses latent-mixture modeling to examine whether learning curves show gradual or stepwise change. The second case study involves crowd-sourced data in which people make predictions about which US National Football League (NFL) players will win the Most Valuable Player (MVP) award. A hierarchical extension to the model is shown to be able to track the current opinion of the crowd, and makes an accurate prediction about the winner.

\section{A Model for Inferring Change}

We present the basic change-point model using some simple illustrative data. After describing the data, we develop the statistical model and detail its software implementation. We then demonstrate the model by applying it to the illustrative data.

\section{Illustrative Data}

Figure 1 show some illustrative data, involving binary responses for a set of $m=3$ items made at $n=20$ discrete sequential times. For the $i$ th item at the $j$ th time $u_{i j}$ and $v_{i j}$ are the number of responses for the two possibilities, with $t_{i j}=u_{i j}+v_{i j}$ being the total number of responses. Each panel corresponds to an item, with circles showing the observed proportion for one alternative over for that item over times. 

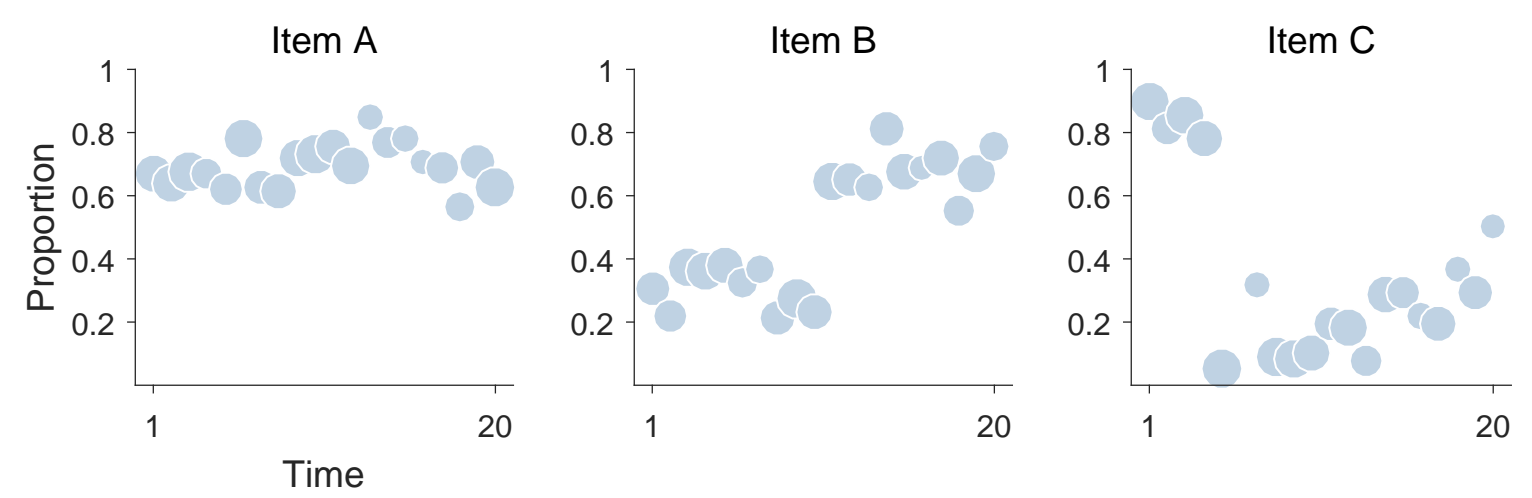

Figure 1. Illustrative binary opinion data for three items, each measured at 20 times. The circles show the proportion of responses for one alternative over the other for each item at each time, and the area of each circle is proportional to the total number of responses at each time.

The area of the circles is proportional to the total number of responses at that time. These simulated data were designed to capture some basic possibilities in step changes over time. The proportion for item $\mathrm{A}$ is always around 0.7 ; item $\mathrm{B}$ changes from 0.3 to 0.7 after the 11 th time period; item $\mathrm{C}$ starts at 0.9 , changes to 0.1 after the 5 th time period; then changes to 0.3 after the 14 th time period. For all three items, the total number of responses varies across the time periods.

\section{Modeling Assumptions}

The core modeling assumptions are most easily introduced in the context of a single item with $u_{j}$ responses for one alternative out of $t_{j}$ total responses for the $j$ th time. It is assumes that there are $\delta<n$ change points $\tau_{1}, \ldots, \tau_{\delta}$ that collectively partition the times into $\delta+1$ stages. The first stage includes all the time periods, $1 \leq j<\tau_{1}$, the second stage includes all the time periods, $\tau_{1} \leq j<\tau_{2}$, and so on, until the final stage $\tau_{\delta} \leq j \leq n$. The stage of at the $j$ th time is $w_{j}=1,2, \ldots, \delta$ and can be determined by counting the number of change points $\tau_{k}$ exceeded by the $j$ th time, so that $w_{j}=\sum_{k} \mathcal{I}\left(j \geq \tau_{k}\right)$, where $\mathcal{I}(\cdot)$ is the indicator function. Once the stage for the at the $j$ th time is determined, the observed responses are simply modeled as $u_{j} \sim \operatorname{Binomial}\left(\theta_{w_{j}}, t_{j}\right)$.

The key to our statistical approach to the change-point inference problem is the prior we employ for the change point parameters. The number of change points $\delta$ and their values $\tau_{1}, \ldots, \tau_{\delta}$ are able to be inferred jointly by using a spike-and-slab 
prior (Mitchell \& Beauchamp, 1988; Rouder, Haaf, \& Vandekerckhove, submitted). These priors are well developed in statistics, and are often useful for simultaneous model selection and parameter inference. Specifically, for each potential change point $k=1, \ldots, \gamma$, we introduce an unordered set of change points $\tau_{k}^{\prime}$, and assign the prior

$$
\tau_{k}^{\prime} \sim \operatorname{Categorical}(\frac{n}{2 n-1}, \overbrace{\left.\frac{1}{2 n-1}, \ldots, \frac{1}{2 n-1}\right)}^{n-1}) .
$$

This categorical prior places a spike at the value $\tau_{k}^{\prime}=1$ and the slab across the remaining times $\tau_{k}^{\prime}=2, \ldots, n$, so that the prior mass given to a change point at the first time is the same as the prior mass given to all subsequent times combined. The insight is that, since inferring a change point at the first time period does not contribute to defining a stage, it corresponds to the inference that the change point does not exist. Thus, there is a prior of one-half for each potential change point that it does not exist. The equal prior mass given to all subsequent change point then corresponds to assuming that, if a change point exists, it is equally likely to occur at any time. Thus, the prior on $\tau_{k}^{\prime}$ simultaneously supports the model selection inference about whether or not a change point exists and the parameter inference about the time of the change conditional on it existing.

The value $\gamma$ is a constant that sets the maximum number of potential change points included in the inference. Conceptually, setting $\gamma$ formalizes prior assumptions about the number of change points. Specifically, since each $\tau_{k}^{\prime}$ has prior probability $\frac{1}{2}$ of corresponding to a change point, the prior probability of $\delta$ change points follows the binomial distribution $\left(\begin{array}{l}\gamma \\ \delta\end{array}\right) \frac{1}{2^{\gamma}}$. Thus, the maximum prior probability will be given to the number of change points being half of $\gamma$, and a good guiding heuristic is to set $\gamma$ to be about twice as large as the number of change points expected.

The final part of the inference is to define the sorted sequence $\tau_{1} \leq \tau_{2} \leq \ldots \leq \tau_{\gamma}$ of the $\tau_{k}^{\prime}$ parameters. This sorting imposes an order constraint to make the model identifiable, so that $\tau_{\gamma}$ is the last potential change point in the time sequence, $\tau_{\gamma-1}$ is the second-last change point, and so on.

\section{Graphical Model}

A graphical model that follows these modeling assumptions, and applies them to a set of items, is presented in Figure 2. Graphical models provide an intuitive 


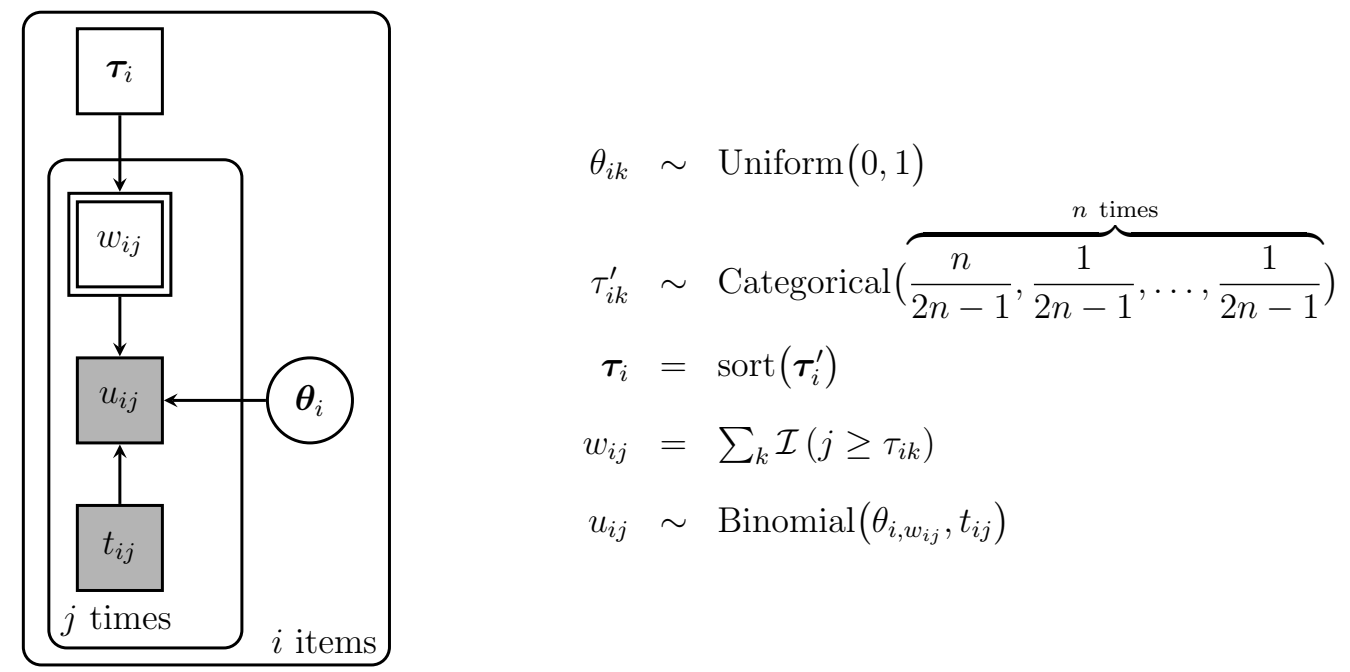

Figure 2. Graphical model representation of the basic change-point model.

formalism for representing probabilistic generative models, and are well suited to the application of computational methods for Bayesian inference (Jordan, 2004; Koller, Friedman, Getoor, \& Taskar, 2007; Lee \& Wagenmakers, 2013). In graphical models, data, parameters, data, and other variables are represented by nodes in a graph, and dependencies between them are represented by the structure of the graph, with children depending on their parents. Different types of nodes are used to indicate what they represent, and we follow the conventions in which observed data or variables are shaded while unobserved parameters are unshaded, continuous values are circular while discrete values are square, and stochastic variables are single bordered while deterministic variables are double bordered. In addition, we use encompassing plates to indicate repetition in the graph structure. The graphical model in Figure 2 has an outer plate for items and an inner plate for times.

The basic version of the model shown in Figure 2 assumes that each stage has the same rate for one response alternative over the other, but that these rates differ independently across stages. Thus, for the $i$ th item, there are change point parameters $\boldsymbol{\tau}_{i}=\left(\tau_{i 1}, \ldots, \tau_{i \gamma}\right)$ and rate parameters $\boldsymbol{\theta}_{i}=\left(\theta_{i 1}, \ldots, \theta_{i, \gamma+1}\right)$ with uniform prior distributions over the range 0 to 1 . The relationship between the time $j$ and the change points determine the stage $w_{i j}$. The response count $u_{i j}$ then depends on the rates, the stage, and the total number of responses $t_{i j}$. The natural generative 
interpretation of the model is that the graphical model shows how the observed counts for a given item at a given time are produced by an underlying stage-dependent rate.

\section{Implementation}

We implement the graphical model in Figure 2 using JAGS (Plummer, 2003), which is standard and free software. JAGS automatically applies computational methods for sampling from the joint posterior distribution of a model, allowing for fully Bayesian analysis. Our implementation involves two JAGS scripts, run sequentially, with the output of the first providing input to the second.

The first JAGS script implements the graphical model in Figure 2 and is listed below. The data are provided in matrices $u$ and $t$ giving the number of responses for one alternative and the total number of responses for each item and each time period. The other inputs are the number of items $\mathrm{m}$, the number of times $\mathrm{n}$, and the maximum number of possible change points gamma.

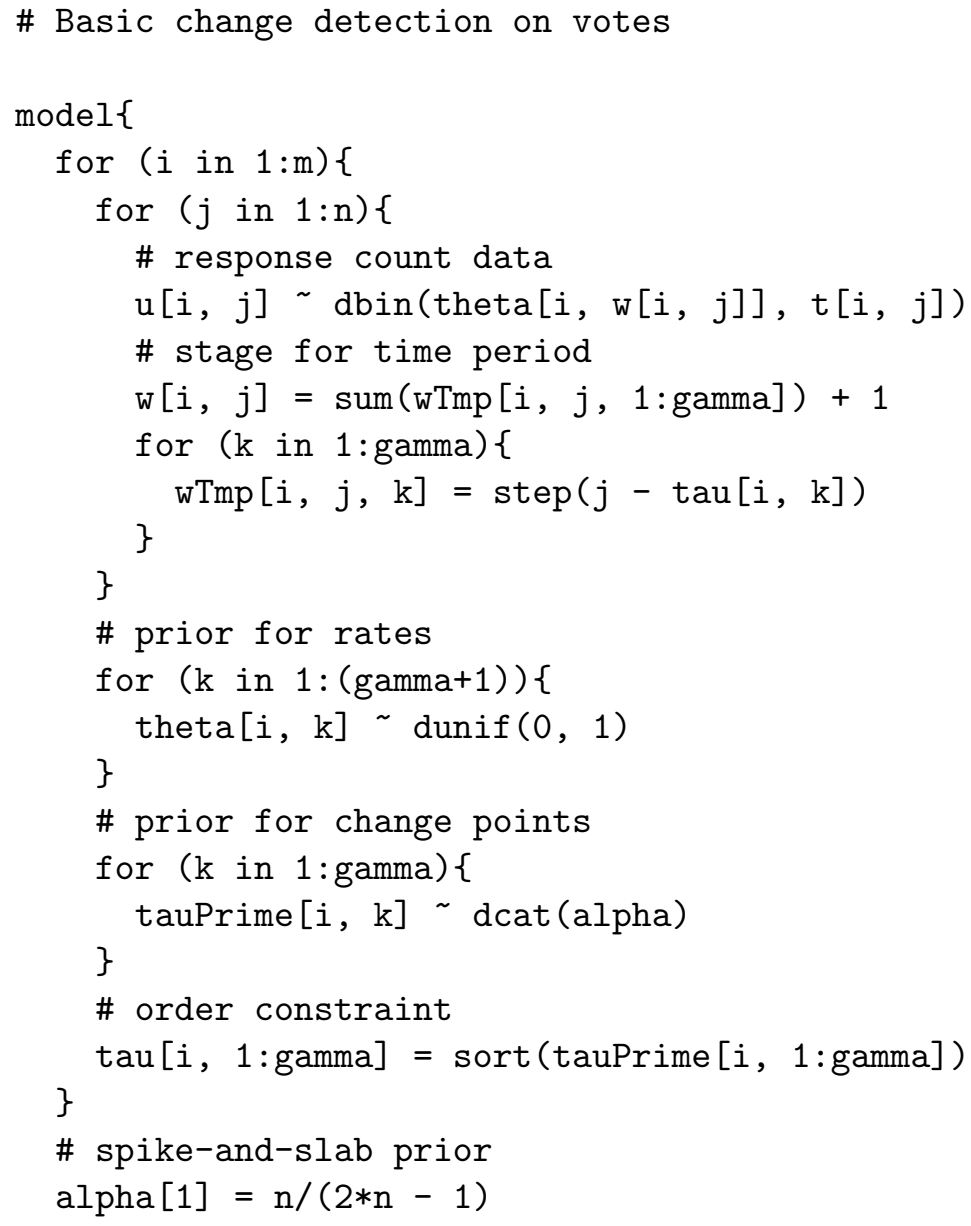




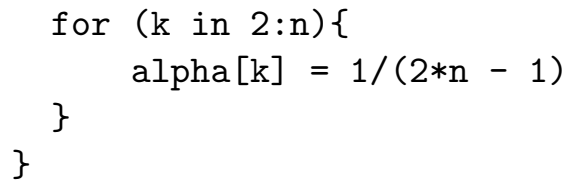

This script follows the graphical model closely. It uses temporary variables wTmp to calculate the stage for each item at each time period, tauPrime to implement the order constraint on the change points, and alpha to define the spike-and-slab prior for the categorical distribution on the change points.

The joint posterior $\boldsymbol{\tau}_{i} \mid \boldsymbol{u}_{i}, \boldsymbol{t}_{i}$ provides the inference about the number and location of the change points for the $i$ th item. Conceptually, the joint posterior is a probability mass distribution over each possible set of change points. There are many ways this joint posterior could be analyzed, but we focus on one approach that emphasizes finding an interpretable set of change points. ${ }^{1}$ This is done by collapsing the uncertainty in the joint posterior, and using the single most likely set of change points. This corresponds to the mode $\boldsymbol{\tau}_{i}^{*}$ of the joint posterior distribution. Inferences about the rates are then be conditioned on this mode, so that the inferred rates correspond to $\boldsymbol{\theta}_{i}^{*} \mid \boldsymbol{\tau}_{i}^{*}$.

It is straightforward to identify the mode of the joint posterior for tau, and then find the posterior distribution for theta from those samples for which tau corresponds to this mode. It is possible, however, that the modal tau is relatively rarely sampled, which means that the posterior distribution would not be well approximated. Thus, our implementation uses the first JAGS script only to find the posterior mode for tau. That mode is then supplied as an observed variable to the second JAGS script listed below.

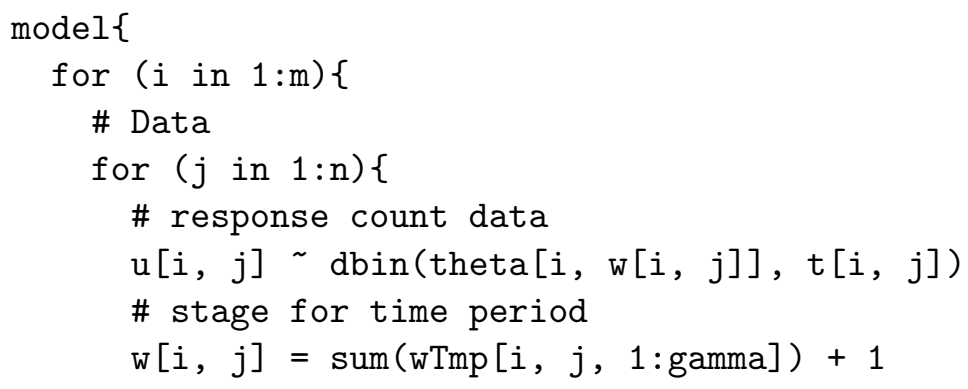

\footnotetext{
${ }^{1}$ If we had different goals, other methods for handling the joint posterior might be appropriate. For example, if our goals focused on the prediction of unseen data, it would be better to use a Bayesian model averaging approach, and incorporate all of the possible sets of change points given non-negligible mass in the posterior.
} 

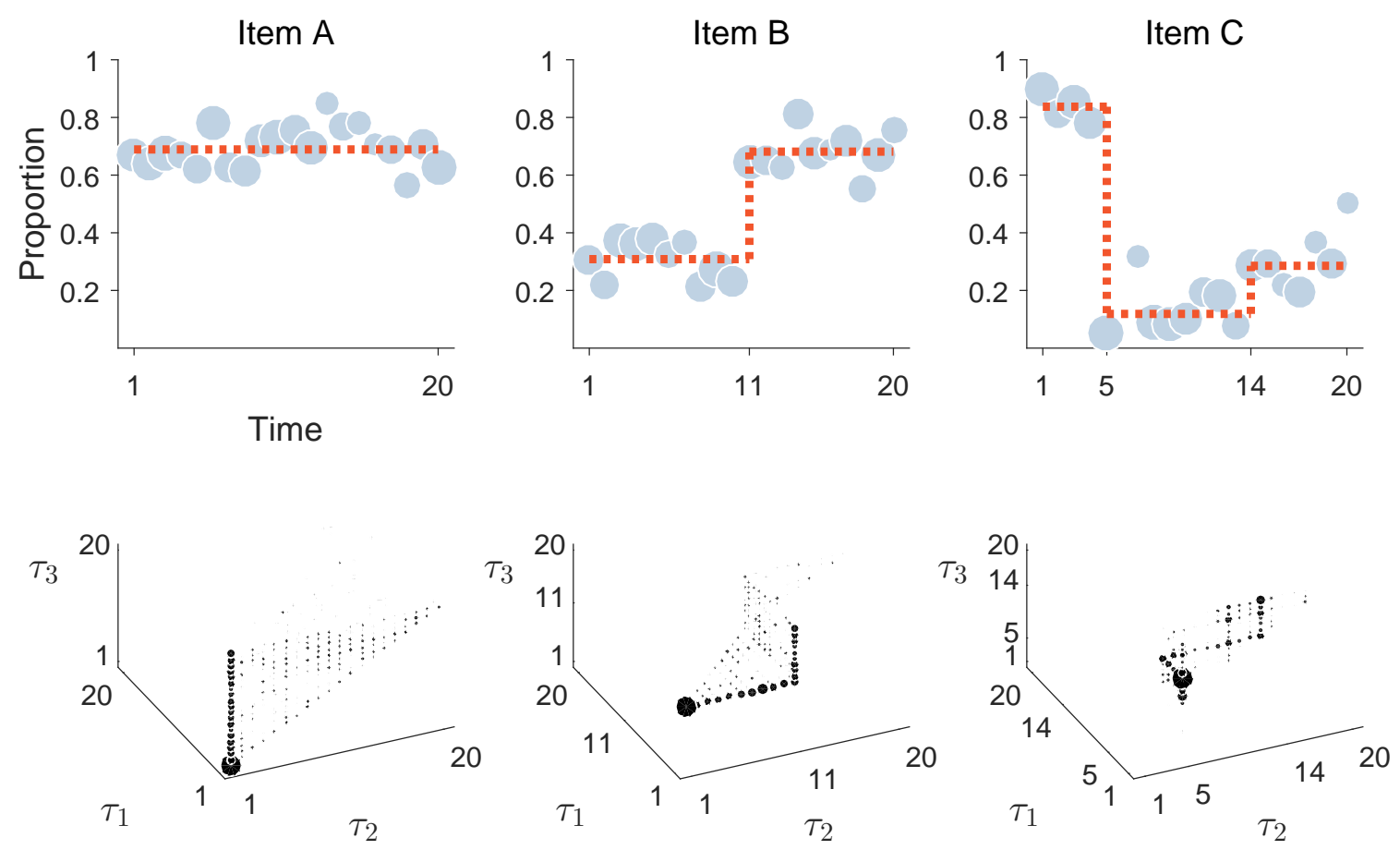

Figure 3. The results of applying the model to the illustrative data. The top panels show the model inferences for each item. The circles show the response proportions for each item at each time period, and the area of each circle is proportional to the total number of responses for the time period. The broken line shows the inferred rate over time, including one change point for item $\mathrm{B}$, and two change points for item $\mathrm{C}$. The bottom panels show the joint posterior distribution for the $\boldsymbol{\tau}$ parameters for each item, the mode of which determines the change points.

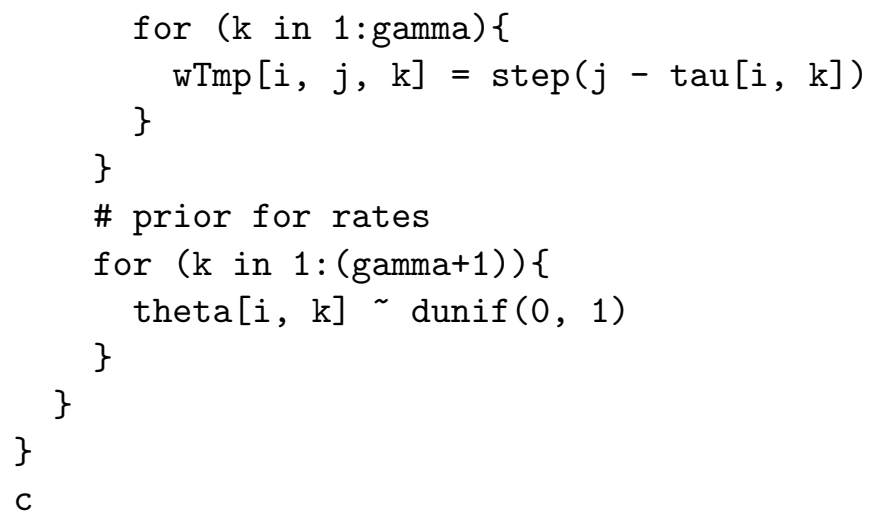

This script is simply a reduced version of the graphical model that assumes tau is known. Accordingly, the inferences for theta are conditioned on the mode for tau. 
Application to Illustrative Data

Figure 3 shows the results of applying the model to the illustrative data. The bottom panels relate to the results of the first step in the implementation, and show the joint posterior $\boldsymbol{\tau}_{i} \mid \boldsymbol{u}_{i}, \boldsymbol{t}_{i}$, based on an upper bound of $\gamma=3$ change points. The resulting three-dimensional joint posterior is shown for each item, with the volume of each cube corresponding to the inferred posterior mass. For item A, the mode is at $\tau_{A}^{*}=(1,1,1)$, corresponding to the inference that there are no change points. For item $\mathrm{B}$, the mode is at $\tau_{B}^{*}=(1,1,11)$, corresponding to the inference that there is a single change point at the 11 th time period. For item $\mathrm{C}$, the mode is at $\tau_{C}^{*}=(1,5,14)$, corresponding to the inference that there are change points at the 5 th and 14 th time periods.

The top panels relate to the results of the second step, in which the rate for each stage is inferred. For each item, the data are again shown by circles, with the inferences about the stages and their rates super-imposed by a broken line. These inferred rates correspond to the posterior expectation of the appropriate rate parameter for each stage. For item A, there is only one stage, and the rate is always about 0.7. For item $\mathrm{B}$, the rate increases from about 0.3 to 0.7 at the change point at the 11 th time period. For item $\mathrm{C}$, the rate falls from about 0.85 to 0.1 at the 5 th time period, then increases to about 0.3 at the 14 th time period. These patterns of change are visually reasonable, given the data, and correspond to the way in which the illustrative data were generated.

\section{Application to Lindisfarne Scribes' Problem}

Blakeley (1949) discusses a philological problem involving the historical use of different $s$-forms for English verbs, such as drives and driveth. The problem revolves around a set of texts known as the Lindisfarne Gospels, which vary in the frequency of their use of these forms. The changes are believed to reflect sequential changes in the scribes of the texts, with each scribe producing some number of consecutive texts before being replaced by the next scribe. Ross (1950) noted that this philological problem can be treated as one of statistical inference, in which the goal is to infer the how many scribes are involved, and which texts they are responsible for, as well as the underlying rate of use of the different $s$-forms for each scribe. Silvey (1958) provides an early attempt to apply statistical methods to make these inference and A. F. M. Smith (1975) provides an impressive early Bayesian approach. 


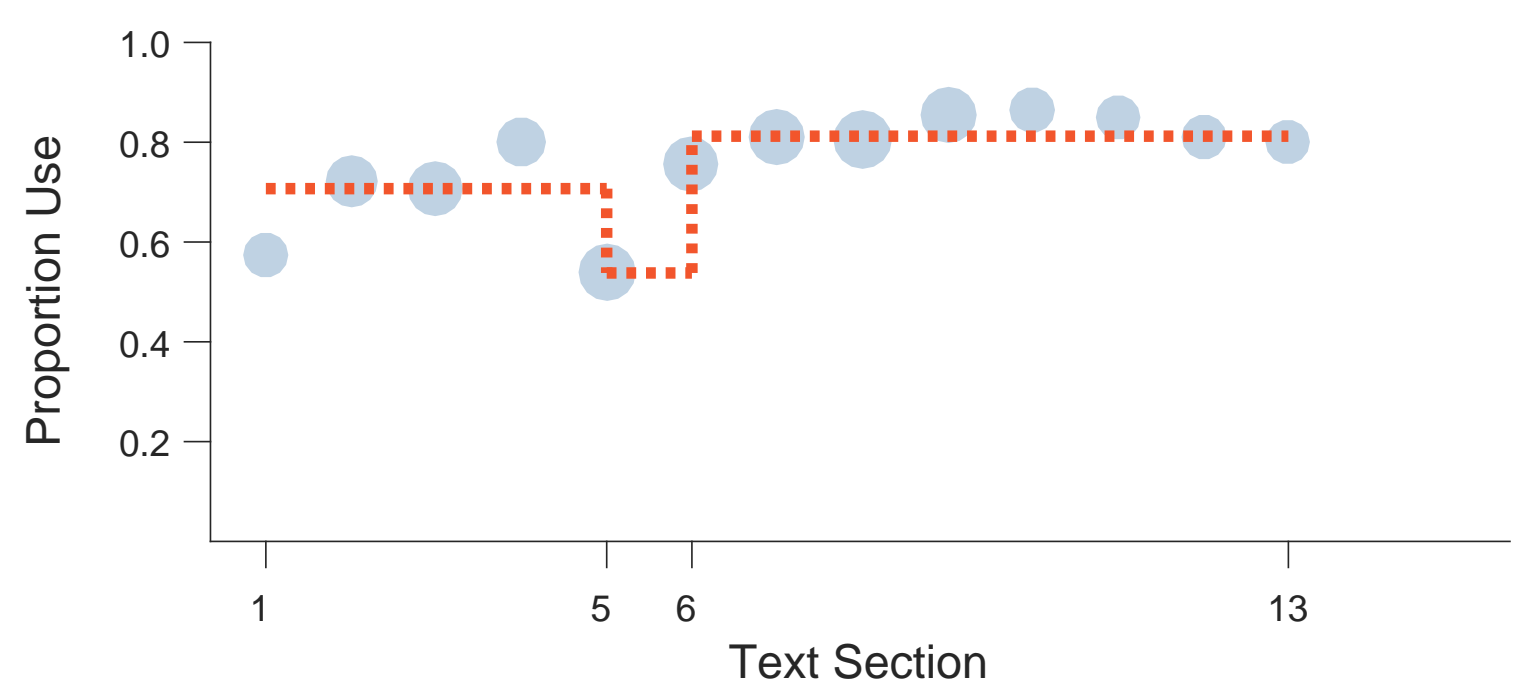

Figure 4. The results of applying the model to a version of the Lindisfarne Scribes data. The circles show the proportion of times one variant form over another for each of the 13 sections of text, and the area of each circle is proportional to the total number of occurrences in that section. The broken line shows the inferred rate of use of the variant form, including change points, interpreted as changes to different scribes, between sections 4 and 5 , and between sections 5 and 6 .

We consider the same data used by A. F. M. Smith (1975, Table 1), which give counts for each form for a sequence of 13 texts. The results of applying the graphical model in Figure 2 are shown in Figure 4. These results are based on setting $\gamma=10$ as an upper-bound on the number of change points. ${ }^{2}$ Two change points are detected after the fourth and fifth texts. The overall inference is that a first scribe produced texts 1-4, a second scribe produced text 5, and a third scribe produced texts 6-13. These results, both in terms of the number of change points and their location, agree with those found by A. F. M. Smith (1975, Tables 4 and 5) using analytic methods.

While it is comforting that our approach produces results that match analytic results for a simple problem, the real advantage of the graphical model implementation is its flexibility. It is straightforward to extend the basic model for inferring change points beyond the case in which there is one fixed binomial rate for each stage. The following two applications aim demonstrate these sorts of expanded modeling

\footnotetext{
${ }^{2}$ Technical details. The JAGS results for this analysis, and all of those that follow, are based on 6 chains, each with 1000 samples, collected after 5000 burn in samples, with thinning every 50th sample. Trace-plots and the standard $\hat{R}$ measure (Brooks \& Gelman, 1997) provided good evidence of convergence.
} 
possibilities, which allow the basic approach to be applied to a broad range of cognitive modeling problems.

\section{Application to Category Learning Curves}

In the first application, we consider human category learning performance. The research question is whether learning curves show gradual improvement or step change increases. This inference problem has basic theoretical implications for the role of two competing accounts of human learning (Ashby \& Maddox, 2005; Kruschke, 2008). Gradual improvement is consistent with various theories of associative learning or reinforcement learning, in which small changes are regularly made to update how stimuli are related to categories. Step change increase is consistent with various theories of hypothesis-testing or rule-based learning, in which new schema are used to determine responding, leading to sudden and potentially large improvements in performance.

Data

We use data from J. D. Smith and Minda (1998, Experiment 3, non-linearlyseparable condition) involving 16 participants learning to categorize 8 nonsense words into two categories based on trial-by-trial feedback. We analyze the learning performance in terms of ten blocks with 56 balanced trials in total, comprised of seven presentations of each of the eight stimuli. Thus, the basic data are, for each participant, a sequence of counts over ten blocks of how many correct responses they made out of 56 trials.

\section{Graphical Model}

Figure 5 shows a latent-mixture model that extends the basic change-point graphical model in Figure 2. There are two components to the latent mixture. One involves step changes in learning performance, with a sequence of increasing probabilities of correct categorization. The other involves a gradual linear increase in performance, starting at chance-level performance, and bounded by perfect performance. The latent binary indicator parameter $z_{i}$ determines whether the learning curve for the $i$ th participant is modeled by the step-change or linear-increase mixture component. The step-change component follows the basic change-point model, inferring how many changes there are in the rate of correct responses, at which blocks 


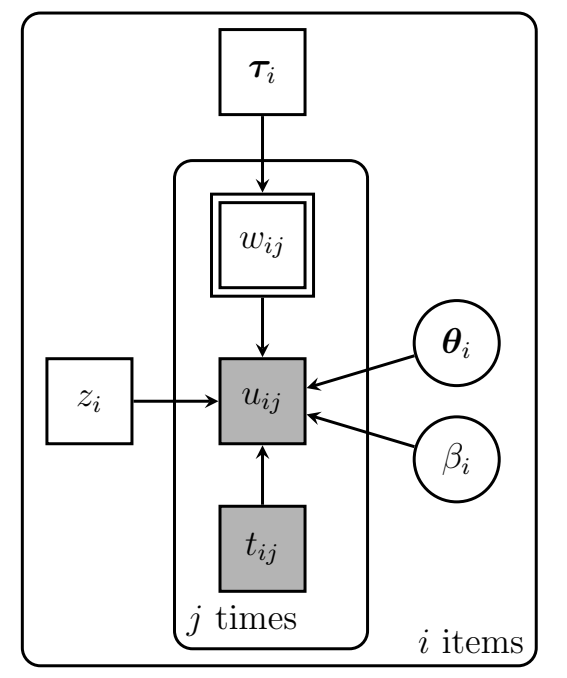

$$
\begin{aligned}
\theta_{i k} & \sim \operatorname{Uniform}(0,1): \theta_{i 1} \leq \theta_{i 2} \leq \ldots \\
\beta_{i} & \sim \operatorname{Uniform}(0,0.5) \\
z_{i} & \sim \operatorname{Bernoulli}\left(\frac{1}{2}\right) \\
\tau_{i k}^{\prime} & \sim \operatorname{Categorical}(\overbrace{\left.\frac{n}{2 n-1}, \frac{1}{2 n-1}, \ldots, \frac{1}{2 n-1}\right)}^{n \text { times }} \\
\boldsymbol{\tau}_{i} & =\operatorname{sort}\left(\boldsymbol{\tau}_{i}^{\prime}\right) \\
w_{i j} & =\sum_{k} \mathcal{I}\left(j \geq \tau_{i k}\right) \\
u_{i j} & \sim \begin{cases}\operatorname{Binomial}\left(\theta_{i, w_{i j}}, t_{i j}\right) & \text { if } z=1 \\
\operatorname{Binomial}\left(\min \left[1, \beta_{i} \times j+0.5\right], t_{i j}\right) & \text { if } z=0\end{cases}
\end{aligned}
$$

Figure 5. Graphical model representation of the latent-mixture extension of the changepoint model.

these changes occur, and what the rate of correct responding is in each stage defined by the change points. The rates are constrained to increase as the stages progress. The linear-increase component assumes that the rate of correct responding begins at 0.5 , and then increases by $\beta_{i}$ over each block, until (possibly) the rate of correct responding is perfect at one. The learning rate $\beta_{i}$ is specific to the $i$ th participant, and is given the prior $\beta_{i} \sim$ Uniform $(0,0.5)$, corresponding to the assumption performance increases over blocks, and can be perfect as early as the second block. The binary indicator variable $z_{i}$ is given the prior $z_{i} \sim \operatorname{Bernoulli}\left(\frac{1}{2}\right)$ corresponding to the assumption that it is equally likely each participant will belong to the step-change or linear-mixture component.

The JAGS script below implements the graphical model in Figure 5. The key change is that $u[i, j]$ now has a binomial rate that depends upon the $z[i]$ latent indicator variable, and a prior is set for each of these indicators in the new \#model indicator section of the script. The linear possibility for the rate is implemented in the new \#linear component section of the script.

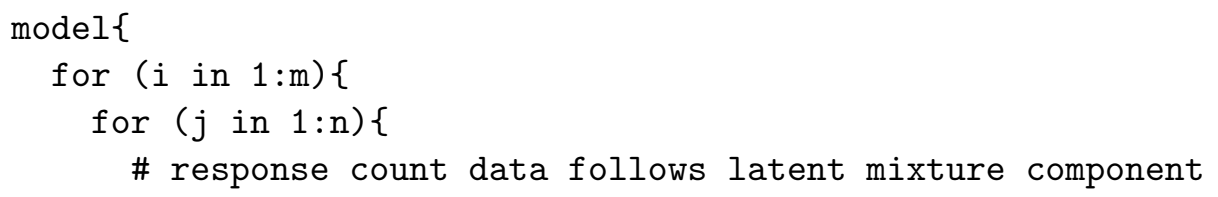




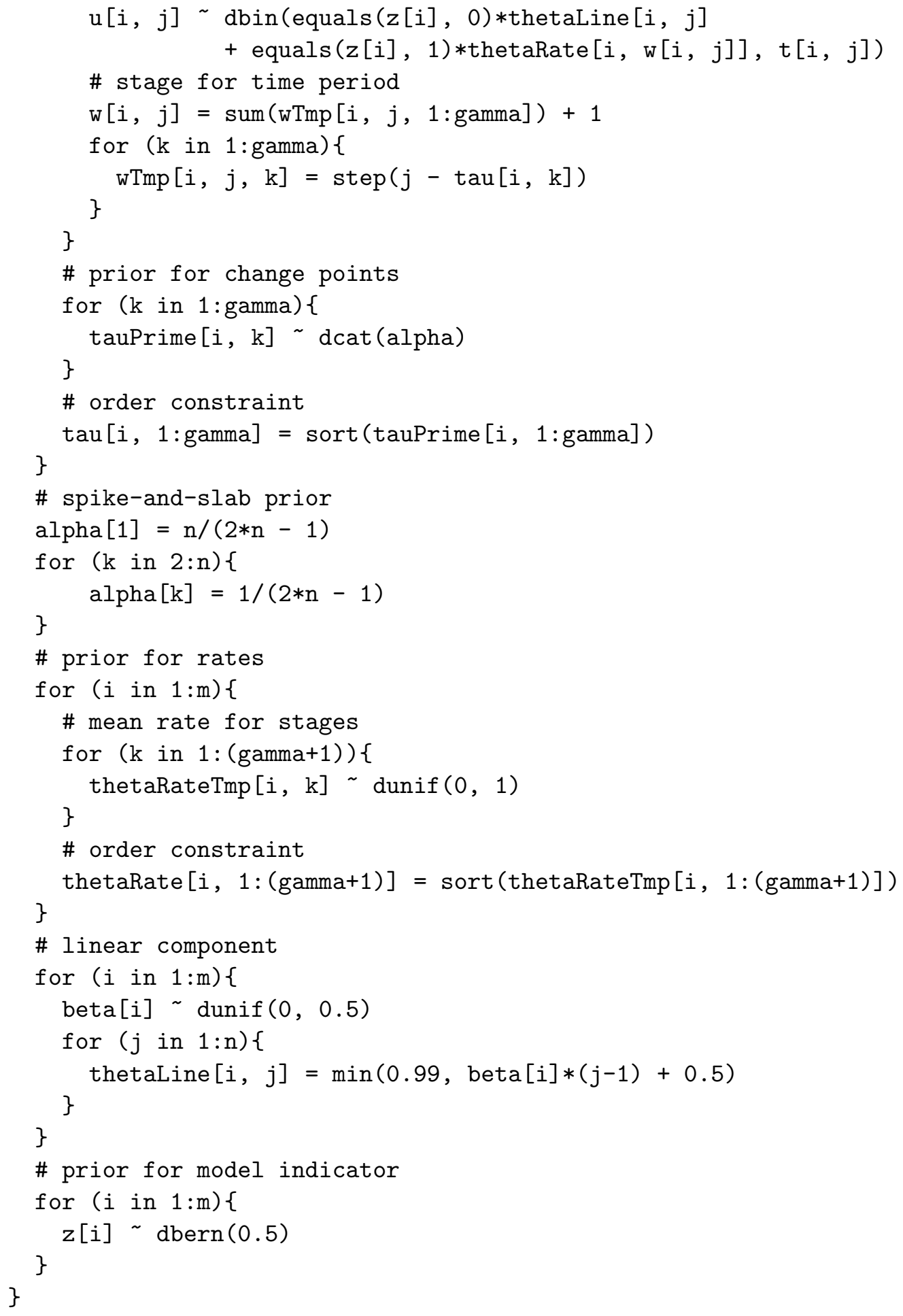

We first use the full joint posterior inferred by this script to find modal latent indicator parameters $\boldsymbol{z}^{*}$. If this mode is $z_{i}^{*}=1$ the participant is classified in the 
step-change mixture component, whereas if the mode is $z_{i}^{*}=0$ the participant is classified in the linear-increase mixture component. For participants in the stepchange component, the mode of the joint posterior $\boldsymbol{\tau}_{i} \mid z_{i}=1, \boldsymbol{u}_{i}, \boldsymbol{t}_{i}$ of their changepoint parameters, conditional on assignment to the step-change component, is found. Both the modal $z_{i}^{*}$ and $\boldsymbol{\tau}_{i}^{*}$ for each participant are then provided as observed values for a second script that infers just the $\boldsymbol{\theta}_{i}$ or $\beta_{i}$ parameters for each participant, depending on their mixture component classification. The second script is not shown, but simply removes the sections that define priors for the $\mathbf{z}$ and tau parameters. It is available as supplementary material.

\section{Modeling Results}

Figure 6 shows the results of applying the latent-mixture model to the J. D. Smith and Minda (1998) data, with the setting $\gamma=5$. The observed proportion of correct categorization decisions for each participant over blocks is shown, together with a summary of the inferences of the model for that participants. If the participant is classified as having step-change learning, the model inferences take the form of a series of mean accuracies for the stages defined by the inferred change points. If the participant is classified as having linear-increase learning, the model inferences are a bounded line based on the learning rate parameter. In each panel, the Bayes factor, derived from the posterior expectation of the $z_{i}$ indicator parameters, is also shown (Lodewyckx et al., 2011). Thus, for example, the Bayes factor for participant 14 is 30 in favor of step-change learning, in which their accuracy improves from about $65 \%$ to about $90 \%$ at the sixth block, while the the Bayes factor for participant 15 is 26 in favor of linear-increase learning, in which their accuracy improves from $50 \%$ to about $90 \%$ over the blocks.

Overall, Figure 6 shows exactly half the participants are inferred to use each of the two learning possibilities. Sometimes the certainty of these inferences, as quantified by the Bayes factor, is relatively weak, especially for participants — such as participants $2,6,13$, and 16 - who show little evidence of learning. This pattern of change in accuracy can be well described by both learning possibilities, but the linear-increase one is generally slightly preferred because it is simpler. For many other

participants, however, there is clear and interpretable evidence for either step-change or linear-increase learning. The learning trajectory for participant 8, for example, shows a clear step-change pattern, with a small increase on the second block, and 

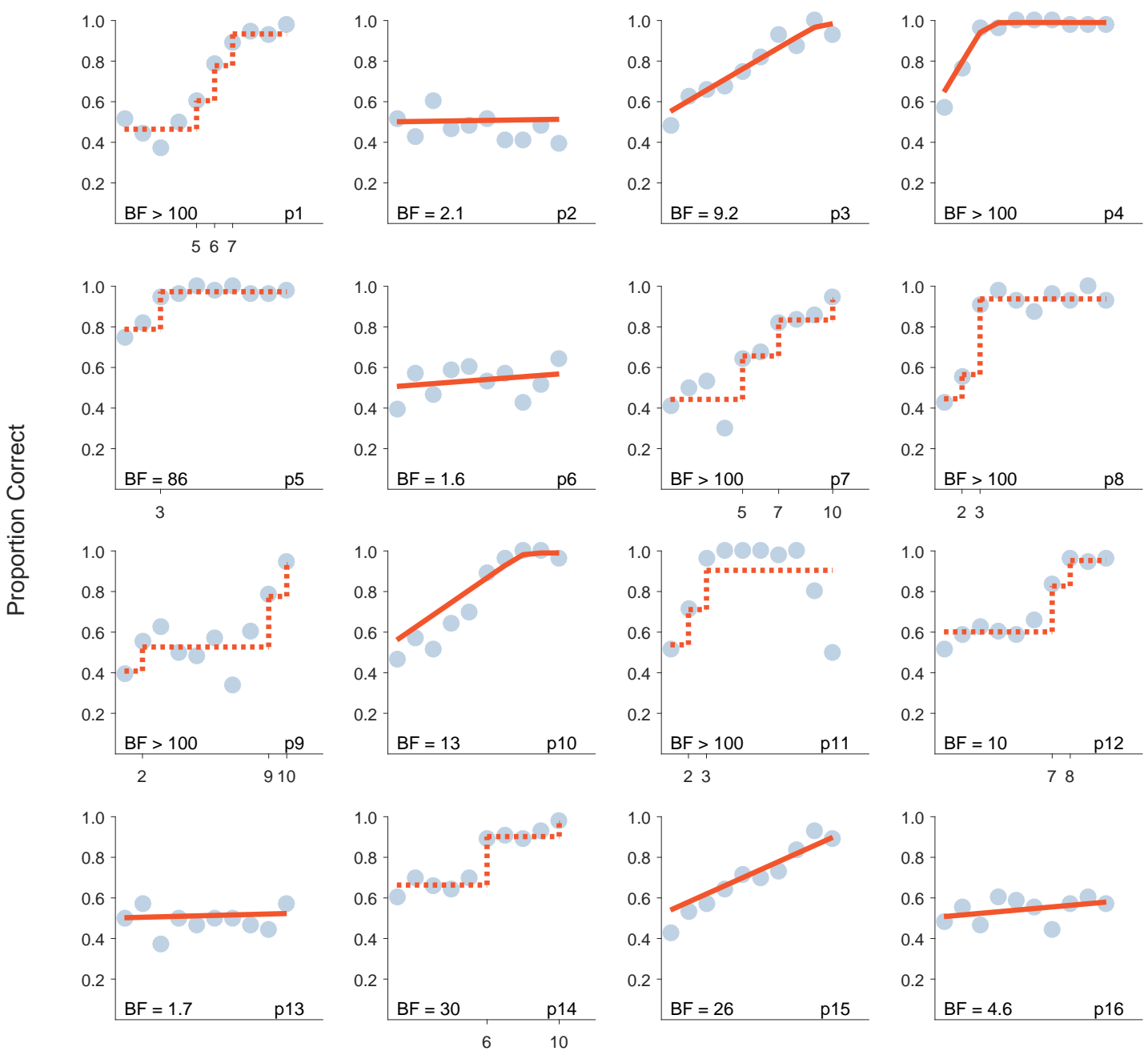

Block

Figure 6. Results of applying the latent-mixture model to the category learning performance reported by J. D. Smith \& Minda (1998, Experiment 3, non-linearly-separable condition). Each panel corresponds to a participant, labeled p1-p16, with circles showing their categorization accuracy over 10 blocks of trials. For participants classified as having step-change performance, a broken line shows the inferred rate of accuracy over each stage, with $x$-axis tick labels detailing the change points. For participants classified as having linear-increase performance, a solid line shows the pattern of change implied by the inferred learning rate. Each panel also presents the Bayes factor (BF) in favor of the inferred classification. 
then a large increase on third block. Similarly, participant 7 shows a clear stepchange pattern. They perform near chance for the first four blocks, improve to about $60 \%$ accuracy for the next two blocks, improve again to about $80 \%$ accuracy for the next three blocks, and finally reach near-perfect accuracy after one more increase on the final block. The learning trajectory for participant 10 shows a steady increase until they reach near-perfect accuracy for the last two blocks. Similarly, participant 4 is inferred to learn with a linear increase, but with a much greater learning rate, reaching near-perfect performance on the third block.

We also applied the model to the other five conditions presented by J. D. Smith and Minda (1998). The results are available as supplementary information, and continue to show evidence for some participants being better modeled by the step-change account of learning than the linearly-increasing one. We do not attempt to draw conclusions about the general prevalence or theoretical importance of step-changes based on these results. Reaching these conclusions would require considering a wider range of alternative models than the simple linear-increasing account used here. What we have achieved, however, is to demonstrate that an account based on step changes can be incorporated into the analysis of category learning curves, using the basic change-point model.

\section{Application to Crowd-Sourced Voting Data}

In the final application, we consider voting data from the crowd-sourced opinion website www.ranker.com. This site consists of tens of thousands of lists, each containing some number of items. Users can rank the items, and can up-vote or down-vote each item at any time. The pattern of voting thus provides a measure of crowd opinion about items, as the opinion evolves over time. We consider voting data for a sporting award - the Most Valuable Player (MVP) of the US National Football League (NFL) — with the goal of detecting changes in the crowd opinion for different players, and using the current opinion for each player at the time the award is determined as a prediction of the winner.

\section{Data}

The data come from the ranker.com list "NFL players most likely to be the 2016-2017 MVP". This list was created on November 18, 2016, and received 31,907 votes for 27 different players, up until Matt Ryan was announced as the winner 
on February 4, 2017. We consider eight players out of the 27, constituting leading candidates for the MVP award. These players all received a large number of votes on the Ranker site's list, and include all of the award favorites discussed in the media.

\section{Graphical Model}

Because the crowd-sourced data involve different people voting at different times, we extend the basic model hierarchically, to allow for variability around a mean group-level rate of positive opinion. Figure 7 shows the hierarchical graphical model. The mean rate for the $x$ th stage is $\mu_{x}$, and the standard deviation for the variation around this mean is $\sigma$. This means that the rate for the $i$ th item at the $j$ th time is $\theta_{i j} \sim \operatorname{Gaussian}_{(0,1)}\left(\mu_{x}, 1 / \sigma^{2}\right)$ where the $j$ th time is in the $x$ th stage. ${ }^{3}$

An assumption of this model is that the variability around the mean is the same for each stage, consistent with the idea that time-to-time fluctuation is a general property of different people expressing opinions at different times, and does not depend on the rate of positive opinion itself, nor on the item being evaluated. Obviously, it would be simple to consider an alternative model in which there is different levels of heterogeneity in the crowd opinion for different stages, different items, or both. The specific prior chosen for $\sigma$ is based on its interpretation as the period-to-period variability in the rate of up-voting that might reasonably be expected. It assumes the standard deviation of this variability follows a positive-truncated Gaussian distribution with a mean of 0 and standard deviation of 0.05. This corresponds to expecting 0-5\% fluctuation over time periods to be common and fluctuations up to $10 \%$ being plausible. Again, it would be simple to consider different assumptions.

The JAGS script below implements the graphical model in Figure 7. The key change arising from the hierarchical extension is the sampling of theta from a Gaussian defined by mu and sigma, and the introduction of priors on mu $[i, j]$ and sigma.

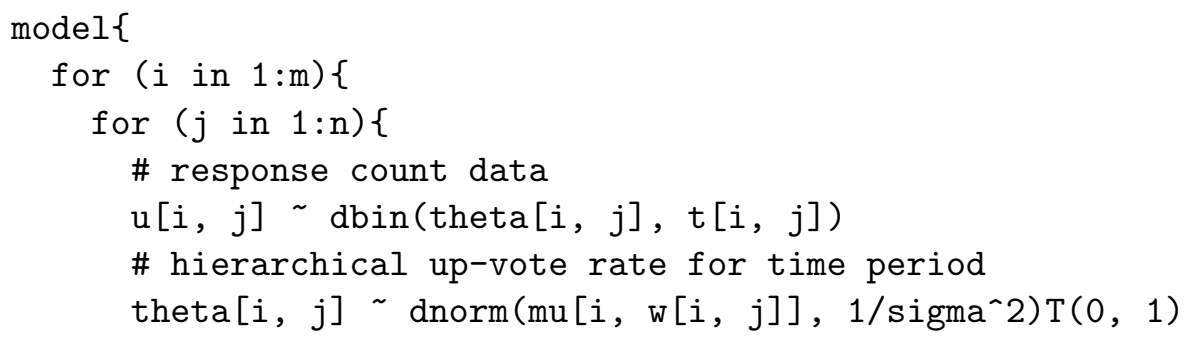

\footnotetext{
${ }^{3}$ This is a truncated Gaussian distribution on the interval $(0,1)$, parametrized in terms of a mean and a precision.
} 


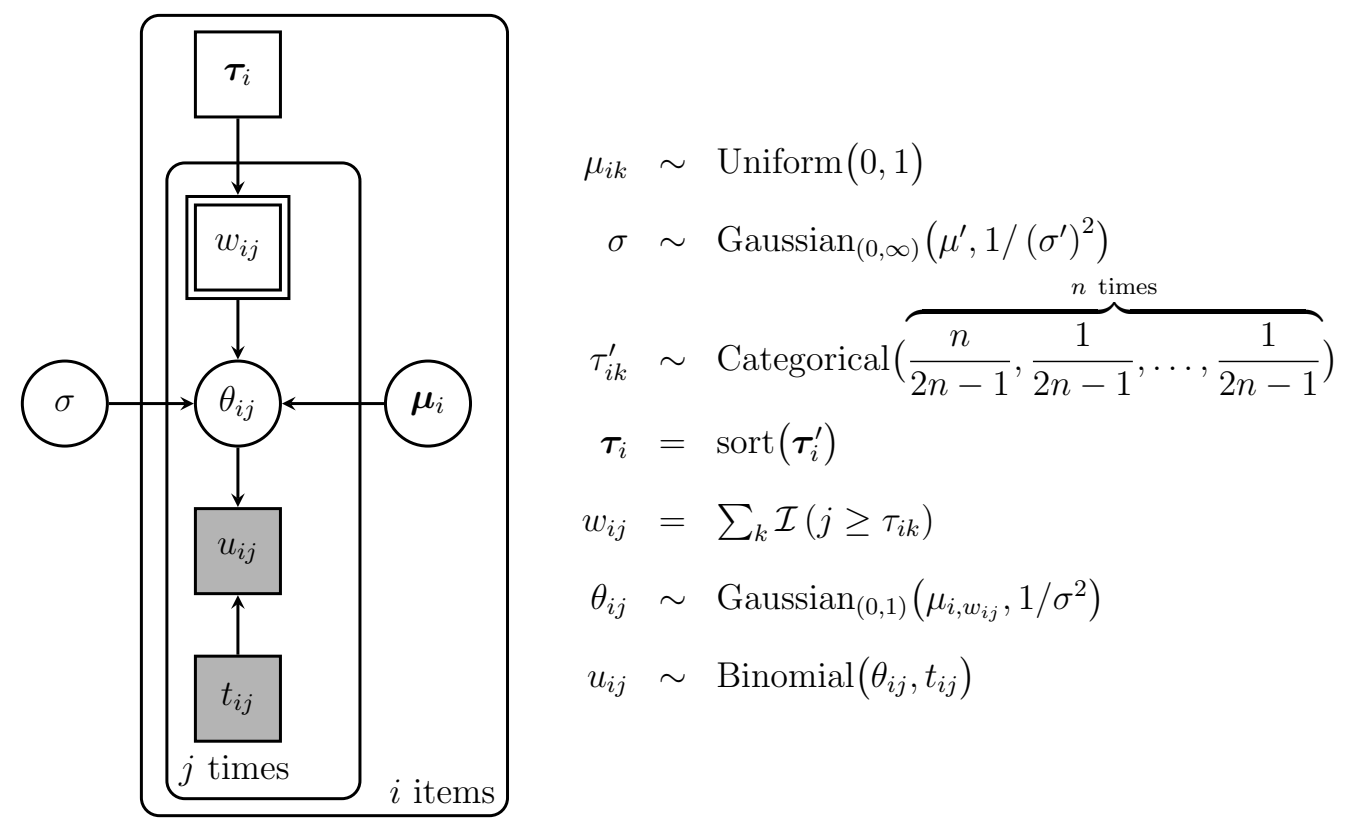

Figure 7. Graphical model representation of the hierarchical extension of the change-point model.

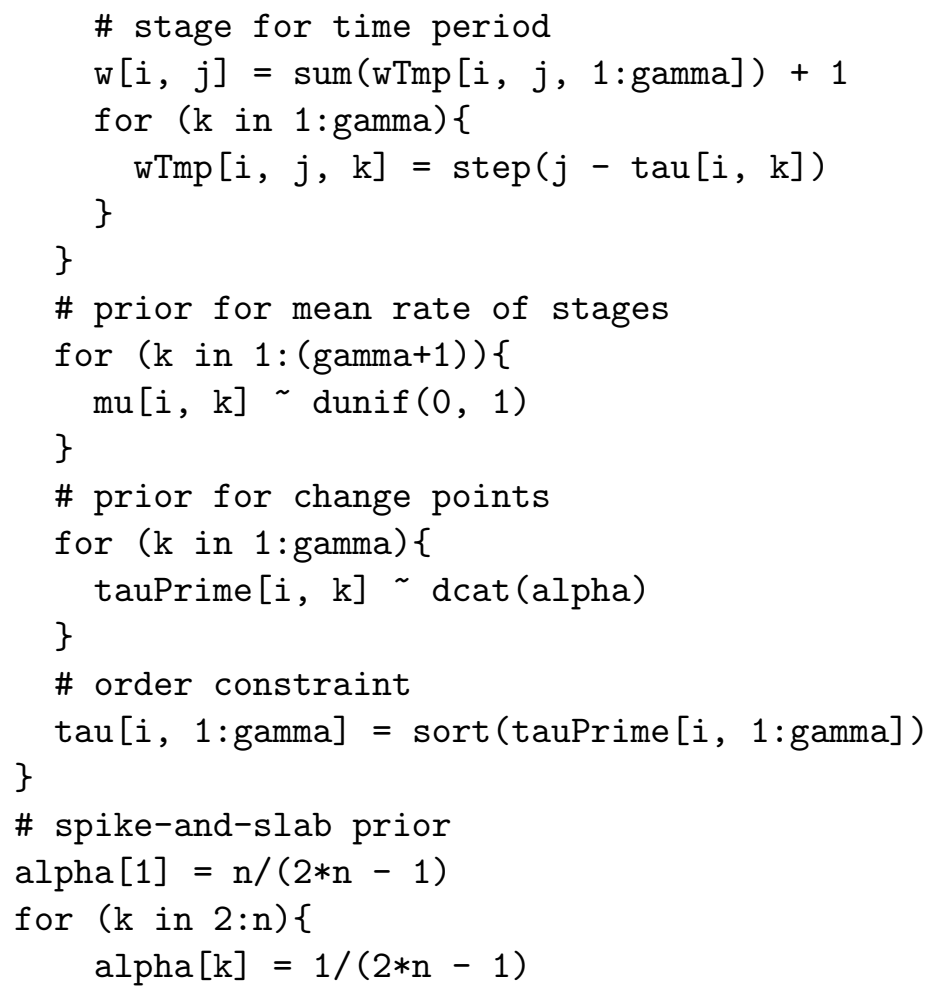




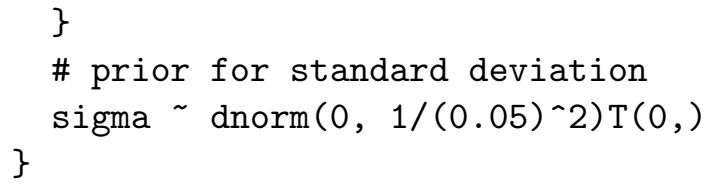

As before, this script is used to find the mode of the change points for each item from the joint posterior. These tau values are used as input for a second script. The second script thus makes inferences about the mean group opinion mu conditioned on the mode for tau. The second script is not shown, but simply removes the sections that define priors for the tau parameters. It is available in the supplementary material.

\section{Modeling Results}

The results of applying the model to these eight players, with the setting $\gamma=5$, are presented in Figure 8. Each panel corresponds to a player and their pattern of votes is shown by circles. The line shows the inferred mean rate of up-voting, with the dates and change points labeled. For example, in the first panel, Aaron Rodgers is inferred to have a mean rate of up-voting of about 35\% until the 3rd of January, when the rate increases to about $60 \%$. There is then a two-day drop to about $10 \%$ from the 23rd to 25th of January, before the rate returns to around $50 \%$ until the final day. The large but brief drop in the rate has a natural interpretation as an immediate overreaction and then re-calibration to Rodgers' Green Bay Packers team's elimination from the playoffs on the 22nd of January.

The inferred changes for the other players in Figure 8 are similarly reasonable, in terms of the voting data, and interpretable, in terms of real-world events affecting their MVP chances. For example, the large drop in the rate of up-voting for Derek Carr occurs at about the time he suffered a season-ending injury. The two increases in the rate of up-voting for Matt Ryan occur immediately after playoff wins for his Atlanta Falcons team, in which he performed especially well as the team's quarterback. Many of the players have an inferred change point on the 3rd of January, immediately after the regular season games were completed. We interpret these changes as the crowd re-evaluating the likely MVP winner at the transition from the regular season to the playoffs.

These data provide a good example of the real-world usefulness of tracking changes in the rate of up-voting. The best prediction of the winner of MVP award 

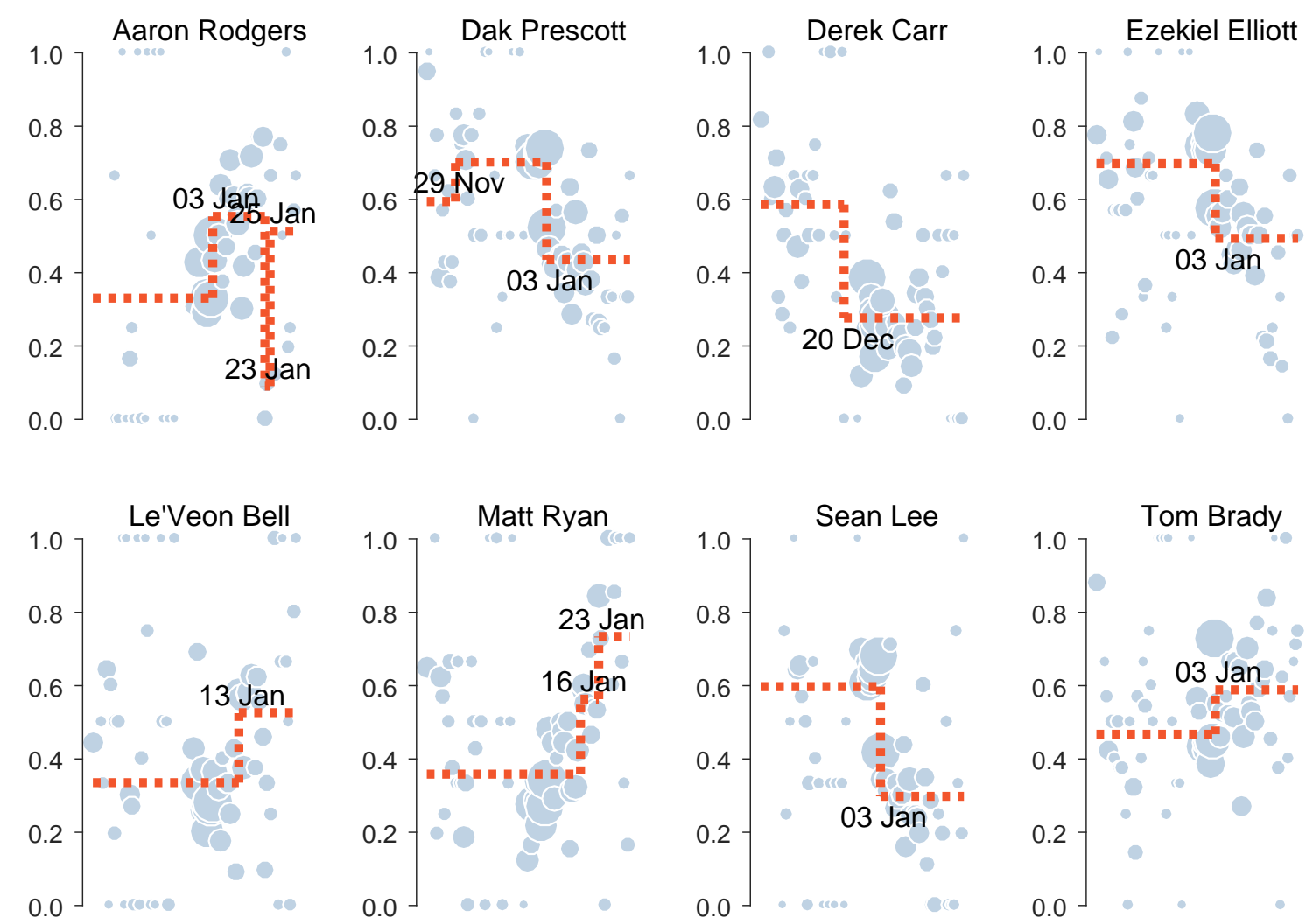

Figure 8. Model results for crowd-sourced binary voting time series data for eight candidates for the NFL MVP award in the 2016-2017 season. Each panel corresponds to a player. The circles show the proportion of up-votes for each item on each day, and the area of each circle is proportional to the total number of votes on that day. The broken line shows the inferred mean rates of up-voting over time, with the date of change points labeled.

is the player with the highest rate at the time the decision is made, at the end of the voting period. If change points are detected in the rate of up-voting the measure of current opinion provided by our model will differ from a standard measure of cumulative opinion that simply measures the overall proportion of up-votes. Formally, the current opinion is the final value of $\mu_{i}$ for the $i$ th player, while their cumulative opinion at the $j$ th time is $\sum_{k=1}^{j} u_{i k} / \sum_{k=1}^{j} t_{i k}$.

Figure 9 shows the cumulative and current opinion for all eight players. The cumulative measure, in the top panel, ranks Ryan in fifth place, behind Ezekiel Elliot, Dak Prescott, Tom Brady, and Aaron Rodgers. Elliot and Prescott were both rookie players for the Dallas Cowboys. and were widely viewed as outstanding new talent in a high-profile franchise that had a historically good regular season. A reasonable 

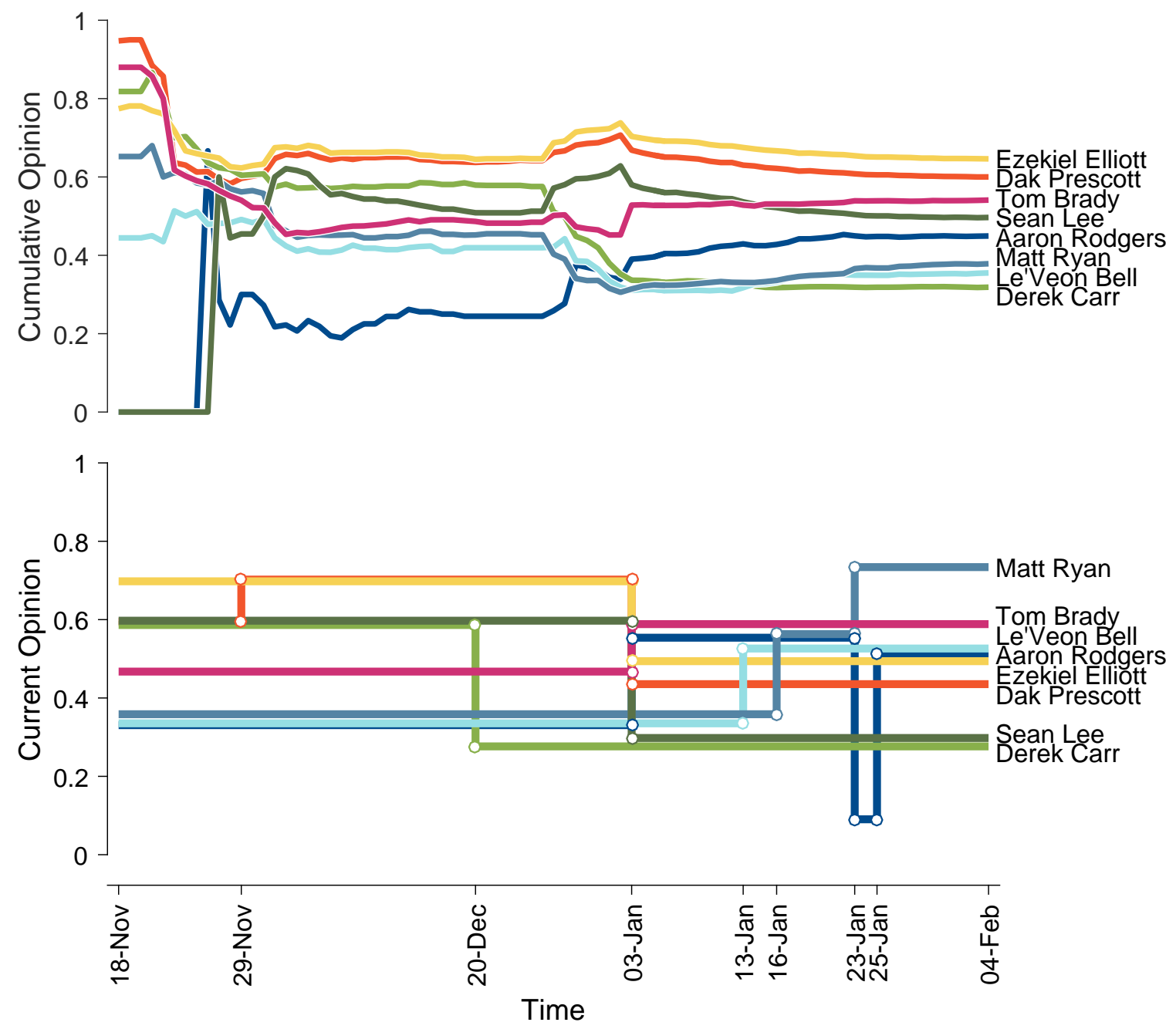

Figure 9. Two analyses of the crowd-sourced voting data for the NFL MVP award in the 2016-2017 season. The top panel shows the cumulative opinion for eight leading candidates. The bottom panel shows the current opinion inferred by the hierarchical change point model, based on the inference of change points in the mean rate of up-voting. Cumulative opinion predicts Ezekiel Elliot as the MVP whereas current opinion correctly predicts Matt Ryan. 
summary of media coverage of their MVP prospects is that early excitement faded to a more realistic assessment that rookie winners are unlikely, especially given both played for the same team. Brady and Rodgers, in contrast, were probably the most established and high-profile perennial favorites for the MVP award. The current opinion measure, in the bottom panel, which is simply the combination of the results for the individual players shown in Figure 8, correctly predicts Matt Ryan as the winner, based on the most recent opinion. It is interesting to note that Brady ranks second according to current opinion, and it is probably fair to speculate he was regarded as the only other serious possible winner in the days before the award was announced.

\section{Discussion}

Sudden changes in measured behavior occur often throughout the cognitive sciences. At the individual level, people can switch their strategies, have sudden insights, or change their opinions. At the group level, different people can be assigned to tasks, or relationships between people can change quickly and drastically. While step change is common, it presents a data analysis and modeling challenge that is not naturally handled by the default statistical methods used in the psychological sciences. Changes that are gradual — such as steady increases, smooth cycles, or random walks - are generally well captured by inherently continuous statistical models like regression, where small changes in inputs lead to small changes in outputs. Modeling step change - requiring identifying where change points lie, and how behavior changes as these points are crossed — presents a different statistical challenge.

In this paper, we have developed and demonstrated a method for modeling step change that fits within a large statistical literature on the topic (Adams \& MacKay, 2007; Barry \& Hartigan, 1993; Chib, 1998; Fearnhead, 2006; Stephens, 1994). A statistical attraction of our approach of the model is that it allows for the number of change points to be inferred together with the locations of the changes. The prior on the latent parameters corresponding to change points combines model selection with parameter estimation, and provides a neat Bayesian solution to the two related inference problems of determining how many change points there are and where they are located.

The statistical approach developed here does require setting a value $\gamma$ that sets the maximum number of change points, and places a prior distribution on the number 
of change points. This part of the model could be extended in two useful ways. One possible extension would be to allow greater flexibility in setting the relative mass of the marginal spike-and-slab priors at the spike that corresponds to the absence of a change point. The current use of one-half is a sensible default, but other possibilities would allow a broader range of prior distributions over the number of change points to be expressed. The other possible extension would be to treat $\gamma$ as a dispersion hyper-parameter, much like non-parametric Bayesian models like Chinese Restuarant Processes (see Navarro, Griffiths, Steyvers, \& Lee, 2006), and make inferences about the number of change points in a more flexible hierarchical setting.

Whatever exact method is used for inferring the number and location of change points, the attraction of our approach lies in its ease of use and flexibility. The core change-point model is implemented as a graphical model using JAGS, and is easily extended within that formalism and software to tackle a diverse range of cognitive modeling problems that involve step change. We presented two worked examples to demonstrate this flexibility: one involving a latent-mixture extension to accommodate change that could be either gradual or sudden, and another involving a hierarchical extension that allowed for homogeneity in the structure of group data to be incorporated.

A final attraction of our approach is more conceptual or philosophical. Our use of generative models places the focus on cognitive assumptions, rather than statistical methods. The extended models of category learning and crowd opinions are built by making assumptions about how the observed behavior is generated over time. Making different or additional cognitive assumptions will lead naturally to a modified or extended model. For example, as alluded to earlier, a category learning theorist might object to the exact assumptions in the linear-increasing account, and propose a different model to compete against the step-change model to explain individual behavior. Almost any model the theorist is likely to propose could be quickly and simply implemented as a graphical model within JAGS (Lee \& Wagenmakers, 2013). Once the generative model is finalized, its application to data is automatically achieved using Bayesian methods. This means that there are no methodological "degrees of freedom" in the way inferences about step change are made. Different models will, of course, lead to different results, but these differences can be understood in terms of the different cognitive assumptions being made. We think this "psychology first" approach to data analysis is a productive one, since it places the emphasis on be- 
ing explicit about the theoretical assumptions being made, and relegates statistical methods to their appropriate service role of specifying the mechanics of inference.

\section{Acknowledgments}

I thank Irina Danileiko for help with the category learning data, Ravi Iyer for help with the ranker .com data, and Lucy Wu for help developing the NFL MVP application. Supplementary material for this paper, including data and code, is available on the Open Science Framework project page https://osf .io/mw3u2/.

\section{References}

Adams, R. P., \& MacKay, D. J. (2007). Bayesian online changepoint detection. arXiv preprint arXiv:0710.3742.

Ashby, F. G., \& Maddox, W. T. (2005). Human category learning. Annual Review of Psychology, 56, 149-178.

Barry, D., \& Hartigan, J. A. (1993). A Bayesian analysis for change point problems. Journal of the American Statistical Association, 88, 309-319.

Blakeley, L. (1949). The Lindisfarne s/ŏ problem. Studia Neophilologica, 22, 15-47.

Brooks, S. P., \& Gelman, A. (1997). General methods for monitoring convergence of iterative simulations. Journal of Computational and Graphical Statistics, 7, 434-455.

Chib, S. (1998). Estimation and comparison of multiple change-point models. Journal of econometrics, 86, 221-241.

Fearnhead, P. (2006). Exact and efficient Bayesian inference for multiple changepoint problems. Statistics and Computing, 16, 203-213.

Green, P. J. (1995). Reversible jump Markov chain Monte Carlo computation and Bayesian model determination. Biometrika, 82, 711-732.

Jordan, M. I. (2004). Graphical models. Statistical Science, 19, 140-155.

Koller, D., Friedman, N., Getoor, L., \& Taskar, B. (2007). Graphical models in a nutshell. In L. Getoor \& B. Taskar (Eds.), Introduction to statistical relational learning. Cambridge, MA: MIT Press.

Kruschke, J. K. (2008). Models of categorization. In R. Sun (Ed.), The Cambridge Handbook of Computational Psychology (pp. 267-301). New York: Cambridge University Press.

Lee, M. D., \& Wagenmakers, E.-J. (2013). Bayesian cognitive modeling: A practical course. Cambridge University Press. 
Lodewyckx, T., Kim, W., Tuerlinckx, F., Kuppens, P., Lee, M. D., \& Wagenmakers, E.-J. (2011). A tutorial on Bayes factor estimation with the product space method. Journal of Mathematical Psychology, 55, 331-347.

Mitchell, T. J., \& Beauchamp, J. J. (1988). Bayesian variable selection in linear regression. Journal of the American Statistical Association(404), 1023-1032.

Navarro, D. J., Griffiths, T. L., Steyvers, M., \& Lee, M. D. (2006). Modeling individual differences using Dirichlet processes. Journal of Mathematical Psychology, 50, 101122.

Pettitt, A. (1979). A non-parametric approach to the change-point problem. Applied Statistics, 126-135.

Plummer, M. (2003). JAGS: A program for analysis of Bayesian graphical models using Gibbs sampling. In K. Hornik, F. Leisch, \& A. Zeileis (Eds.), Proceedings of the 3rd International Workshop on Distributed Statistical Computing. Vienna, Austria.

Ross, A. S. (1950). Philological probability problems. Journal of the Royal Statistical Society. Series B, 19-59.

Rouder, J. N., Haaf, J., \& Vandekerckhove, J. (submitted). Bayesian inference for psychology, part IV: Parameter estimation and Bayes factors. Manuscript submitted for publication.

Silvey, S. (1958). The Lindisfarne scribes' problem. Journal of the Royal Statistical Society. Series B, 93-101.

Smith, A. F. M. (1975). A Bayesian approach to inference about a change-point in a sequence of random variables. Biometrika, 62, 407-416.

Smith, J. D., \& Minda, J. P. (1998). Prototypes in the mist: The early epochs of category learning. Journal of Experimental Psychology: Learning, Memory and Cognition, 24, 1411-1436.

Stephens, D. (1994). Bayesian retrospective multiple-changepoint identification. Applied Statistics, 159-178.

Venter, J., \& Steel, S. (1996). Finding multiple abrupt change points. Computational Statistics \& Data Analysis, 22, 481-504. 